\title{
Molecular Dynamics Study of Implant and Damage Formation in Low-Energy Boron Cluster Ion Implantation
}

\author{
Takaaki Aoki, Jiro Matsuo, Zinetulla Insepov and Isao Yamada \\ Ion Beam Engineering Experimental Laboratory, Kyoto University \\ Sakyo, Kyoto, 606-8501, JAPAN \\ t-aoki@kuee.kyoto-u.ac.jp
}

\begin{abstract}
Cluster ion implantation using decaborane $\left(B_{10} H_{14}\right)$ has been proposed as a useful technique for shallow junction formation. In order to examine the characteristics and advantages of cluster ion implantation, molecular dynamics simulations of small $B$ cluster and monomer implantation were performed. $B_{1}, B_{4}$ and $B_{10}$ are irradiated on $S i(001)$ substrates with acceleration energy of $230 \mathrm{eV} /$ atom so that $B_{4}$ and $B_{10}$ are accelerated with $0.92 \mathrm{keV}$ and $2.3 \mathrm{keV}$, respectively. Those three show the same implant profile and implant efficiency, which agrees with the experimental result of $B_{10} \mathrm{H}_{14}$ implantation. This result suggests that each $B$ atom in a $B$ cluster acts individually in similar way to a monomer ion. B clusters show the same properties in projection range and implant efficiency as the monomer whereas non-linearity is shown in damage formation. The number of displacements by one $B$ atom once increases to the same maximum value for both a $B$ cluster and a $B$ monomer. However, the damage recovery process is different depending on the cluster.size. Damage induced by $B_{10}$ recovers more slowly and 4 times as many displacements remain compared to $B_{1} 8 \mathrm{ps}$ after impact. These displacements by $B_{10}$ clusters concentrate in the near surface region of the impact point, while the ones by $B_{1}$ reside around the implanted $B$ atom as the end-of-range damage. This characteristic damage formation by $B_{10}$ cluster is expected to avoid transient-enhanced-diffusion of incident $B$ atoms and achieve the formation of high-quality shallow p-type junction.
\end{abstract}

\section{INTRODUCTION}

As the scale of LSI device decreases, the formation of high-quality shallow p-type junction becomes more important. In order to fabricate a sub- $0.1 \mu \mathrm{m}$ p-MOS device, boron atoms are expected to be implanted with less than $1 \mathrm{keV}[1,2]$. However, as the energy of implant energy goes down, it becomes more difficult to obtain enough current for industrial fabrication because of space charge effect [3]. Furthermore, the Transient Enhanced Diffusion (TED) of dopant boron atoms becomes a serious problem as the incident energy decreases [4]. The boron cluster ion implantation technique using decaborane $\left(\mathrm{B}_{10} \mathrm{H}_{14}\right)$ has been proposed as one solution for shallow junction formation $[5,6]$. It has been experimentally observed that the implant range of $\mathrm{B}$ atoms using the $\mathrm{B}_{10} \mathrm{H}_{14}$ ion implantation is equivalent to that of monomer $B$ ions accelerated with $1 / 10$ of the energy of $B_{10} H_{14}$ [5]. $\mathrm{B}_{10} \mathrm{H}_{14}$ implantation has great advantages in shallow junction formation because no extra acceleration and decceralation system is needed and charges on the wafer, which cause destruction of devices, is reduced to $1 / 10$ compared with monomer ion implantation. There are other advantages, cluster ion implantation is expected to have a non-linear effect caused by the high-density irradiation of incident atoms. As indicated in earlier work with Ar cluster and fullerene by both molecular dynamics simulations and experimentally, the collisional process of a large cluster is far different from that of monomer ion [7-9]. When a large cluster impinges into the substrate, several times larger displacements are created compared to monomers with same incident energy per atom. $\mathrm{B}_{10} \mathrm{H}_{34}$ should be considered as a material on the border between cluster and monomer and, therefore, it is important to examine the similarity and differences between $\mathrm{B}_{10} \mathrm{H}_{14}$ and $\mathrm{B}_{1}$ ion implantation. In this paper, molecular dynamics simulations of small boron cluster and boron monomer implanting into Si (001) substrate were performed and the advantages of cluster ion implantation technique are discussed.

\section{Simulation METHOD}

In order to examine the implant process of small boron clusters, the molecular dynamics (MD) simulation of $B_{1}, B_{4}$ and $B_{10}$ monomer/cluster impacting on a Si (001) substrate are performed. The Stillinger-Weber potential model [10] is
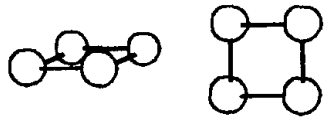

Horizontal
Square

(B4 Horz. Sq.)
Vertical Square (B4 Vert. Sq.)

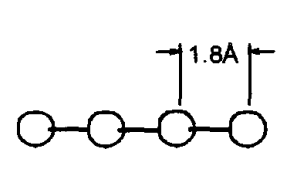

Horizontal

Chain

(B4 Horz. Ch.)
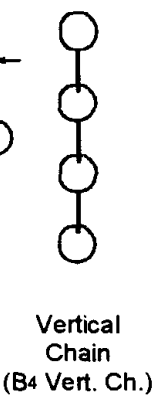

(B4 Vert. Ch.)

B4 cluster

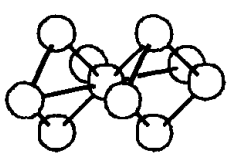

Spherical

(B10 Sphr.)

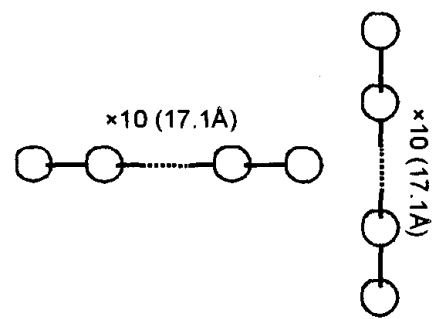

Horizontal

Chain

(B10 Horz. Ch.)
Vertical

Chain

(B10 Vert. Ch.)

B10 cluster

Fig. 1: Structures and orientations at impact of $B_{4}$ and $B_{11}$ clusters prepared for this work. 
applied to the inter-atomic potential of $\mathrm{Si}-\mathrm{Si}$ and the $\mathrm{ZBL}$ model [11] is applied to B-B and B-Si. A Si (001) substrate is considered consisting of 32768 atoms with a cube side of about $90 \AA$. Periodic boundary conditions are applied to the $(100)$ and $(010)$ boundaries and the atoms in lowest layer are fixed to retain the bulk structure. Before implantation of a B monomer/cluster, the substrate is heated to $300 \mathrm{~K}$ and the top surface is reconstructed to $2 \times 1$ structure. In this work, $B_{4}$ and $B_{10}$ are irradiated with a different geometry as shown in Fig. 1 . Because, as the cluster size decreases, the structure and orientation of the cluster at impact becomes more significant, whereas large clusters have a spherical structure and no dependency of orientation. In the case of $B_{4}$, two cluster structures were considered; one has a square structure and the other a chain structure, and each $B_{4}$ cluster impacts parallel and perpendicular direction to the $\mathrm{Si}$ surface. $A B_{10}$ cluster is implanted as a horizontal chain, a vertical chain and a spherical f.c.c. structure. The distance between the $B$ atoms is $1.8 \AA$ for each structure which was calculated from the atomic radius of a boron atom. $B_{1}$ monomer and vertical $B_{4}$ and $B_{10}$ chains are implanted with an incident angle of $7^{\circ}$ to the surface normal and rotated $30^{\circ}$ to the $\{001\}$ direction to avoid channeling implantation, and other clusters are implanted with normal direction. In order to obtain statistical properties, such as depth profile of implant atoms and displacements, 100 simulations for $B_{1}$ and 25 simulations for both $B_{4}$ and $B_{10}$ were done at different impact points, respectively.

\section{RESULTS AND DISCUSSION}

\section{A. Implant Efficiency and Profile of Boron Atoms}

Figs. 2(a)-2(c) show the depth profiles of implanted $B$ atoms from $B_{1}, B_{4}$ and $B_{10}$, respectively, $1.2 p s$ after impact. The region on the RH side where the depth is larger than $0 \AA$ indicates the substrate and the values on the LH side indicate the ratio of unimplanted boron atoms to the total irradiated atoms. $B_{1}, B_{4}$ and $B_{30}$ are irradiated on the target with the same incident energy of $230 \mathrm{eV} / \mathrm{atom}$ so that the total incident energy of $B_{4}$ and $B_{10}$ is $0.92 \mathrm{keV}$ and $2.3 \mathrm{keV}$, respectively. $B_{4}$ and $B_{10}$ implantation shows the same implant efficiency as $B_{1}$ of about $82 \%$, except for the case of the vertical $B_{4}$ and $B_{10}$ chains. In these cases, almost all $B$ atoms are implanted into the substrate without all colliding with the substrate atoms. It is considered that the first B atom of a chain cluster knockson a substrate $\mathrm{Si}$ atom and the following $\mathrm{B}$ atoms can penetrate to deeper regions. However, the implant efficiency of square $B_{4}$ clusters shows a similar value as that of $B_{1}$ and the horizontal $B_{4}$ chain cluster. This result suggests that the stack number of two in a $B_{4}$ square is not enough density to cause an improvement in implant efficiency, but four is enough for this incident energy of $230 \mathrm{eV} / \mathrm{atom}$. This assumption is supported by the spherical $B_{10}$ in which the stack number is two or three and shows similar implant efficiency to that of a horizontal $B_{10}$ chain rather than a vertical $B_{10}$ chain. From the viewpoint of the implant profile, there is no significant difference among the $B_{4}$ clusters, almost all $B_{10}$ and the $B_{1}$ monomer, but only the vertical $B_{10}$ chain cluster shows a deeper profile compared with the other implant profiles. In the case of the vertical $B_{4}$ chain cluster impact, the $\mathrm{B}_{4}$ cluster collapses immediately after it penetrates the first layer of the substrate. However, the $B_{10}$ chain keeps

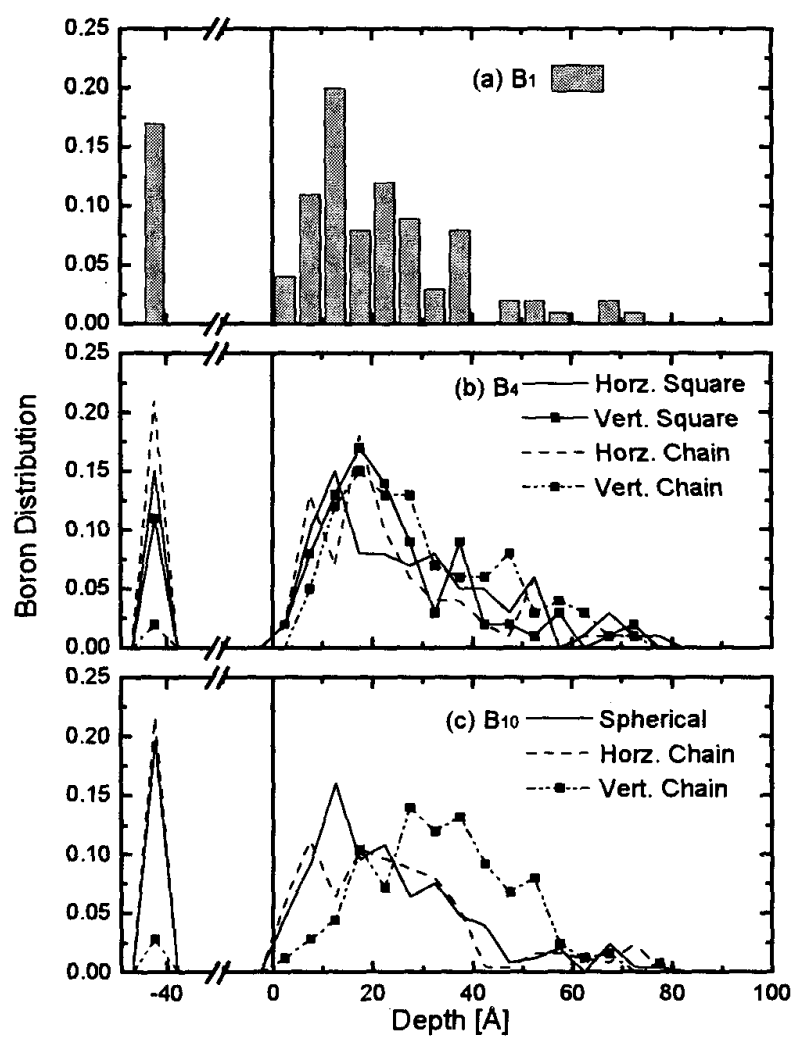

Fig. 2: Implant profile of $B$ atoms by (a) $B_{1}$, (b) $B_{4}$ and (c) $B_{10}$ implantation. The depth of $O A$ shows the surface of the substrate and the values on the left side indicate the ratio of unimplanted $B$ atoms to total irradiated atoms. Note that the ratios of unimplanted atoms by vertical chain-like $B_{4}$ and $B_{10}$ are reduced.

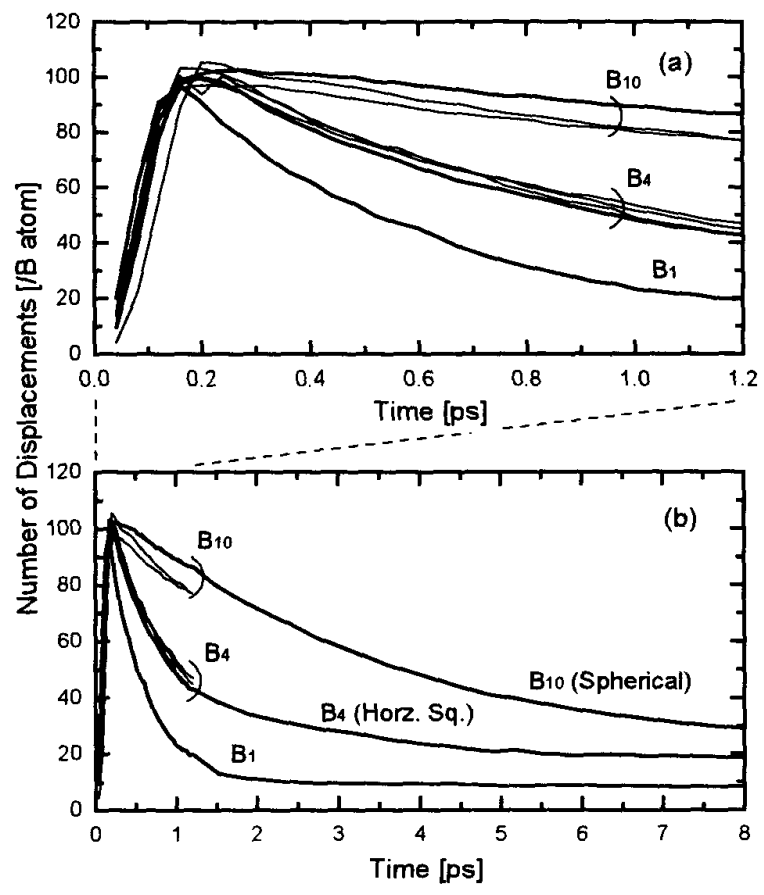

Fig. 3: Time transition of the number of displacements induced by boron monomer and clusters. Fig. 3(a) shows the results for every variation of monomer/cluster until $1.2 \mathrm{ps}$ and Fig. $3(\mathrm{~b})$ shows the following results of $B_{1}$, horizontal $\mathrm{B}_{4}$ square and spherical $\mathrm{B}_{10}$ cluster until $8 \mathrm{ps}$. 


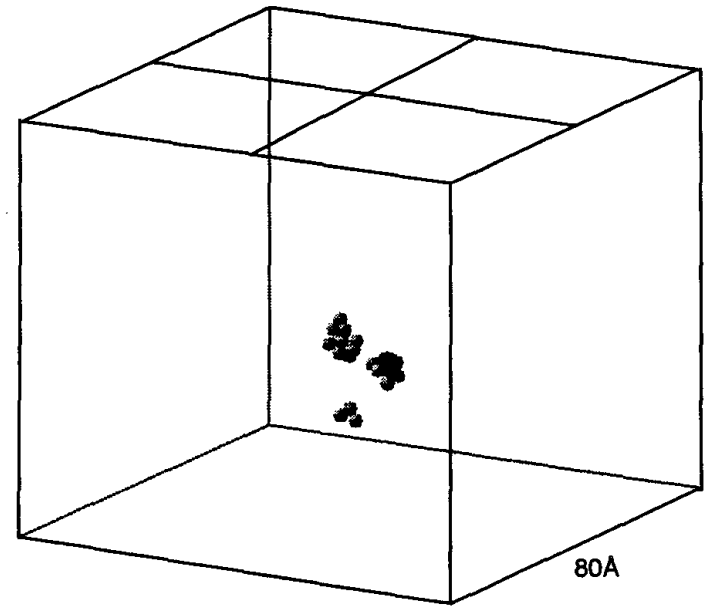

B1

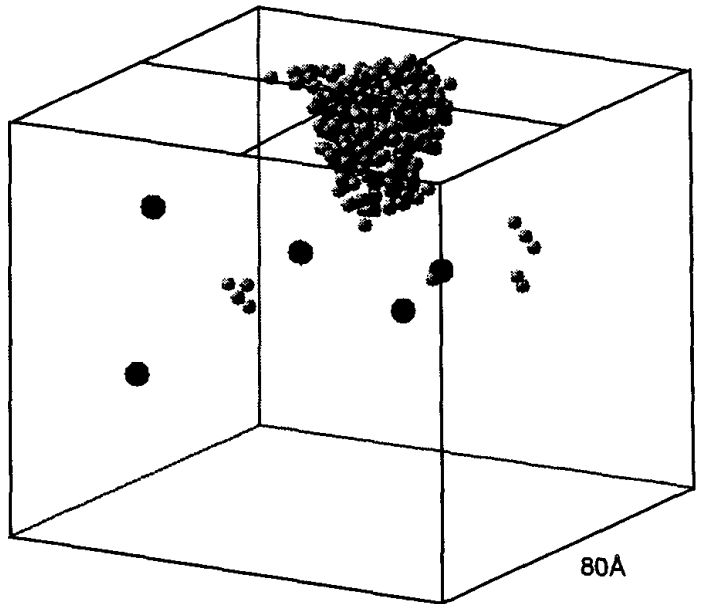

B10 Spherical Structure

Fig. 4: Snapshots of $B_{1}$ monomer and $B_{10}$ cluster implanted with 230eV/atom, 8ps after impact. Black spheres represent the implanted $B$ atoms and gray ones are displaced Si atoms.

the coherency of velocity in the substrate and each B atom continues to penetrate deeply into the substrate. When the interaction between the incident $B$ atoms is less probable, as in the cases of a chain or a spherical $B_{10}$ cluster, each $B$ atom in the cluster acts in a way similar to monomer ions with the same energy per atom. The result of implant efficiency and implant profiles are in agreement with the experimental results that $\mathrm{B}_{10} \mathrm{H}_{14}$ which has spherical structure and shows the same implant profile as $B$ monomer ions accelerated with $1 / 10$ of the energy of $\mathrm{B}_{10} \mathrm{H}_{14}[5]$. These experimental and MD results demonstrate that each $B$ atom in $B_{10} \mathrm{H}_{14}$ can be treated as independent $\mathrm{B}$ monomer ions.

\section{B. Damage Formation by Boron Cluster Impact}

Fig. 3(a) and Fig. 3(b) show the time dependence of the number of displacements per single $B$ atom. The displacements are defined as the $\mathrm{Si}$ atoms which have a potential energy of $0.4 \mathrm{eV}$ above the bulk state [12]. Fig. 3(a) shows the transition of the displacement yield by $B_{1}$ monomers and various $B_{4}$ and $B_{10}$ clusters from $0 \mathrm{ps}$ to $1.2 \mathrm{ps}$. Fig. 3(b) shows the results from the calculation continued on to 8 ps for $B_{1}$, horizontal $B_{4}$ square and spherical $B_{10}$. Until the time of $0.2 \mathrm{ps}$, almost all of the incident energy from initial $B$ atoms is transferred to substrate atoms, and the number of displacements reaches the maximum value at around $0.2 \mathrm{ps}$ and then decreases. The maximum number of displacements depends the neither on cluster size nor on cluster structure. It is considered that each implanted B atom interacts with the substrate atoms individually and the kinetic energy of the incident atoms is transferred to the substrate without overlapping. This suggestion agrees with the aforementioned result that implant profile and implant efficiency does not have size and structure dependency except for the case of the vertical chain cluster. The damage recovery process is different depending on the cluster size. As shown in Fig. 3(b), the displacements induced by $B_{1}$ recover rapidly in $2 p s$ and about eight displacements remain 8ps after impact. However, the damage recovery speed becomes slower as the cluster size increases. In the case of spherical $B_{10}$ impact, about 30 displacements, which is four times higher than $B_{1}$, remain $8 \mathrm{ps}$ after impact. The high yield displacement by cluster ion impact is due to the high-density energy irradiation effect. When a $B_{10}$ cluster impacts the substrate, $B_{10}$ deposits its incident kinetic energy of $2.3 \mathrm{keV}$ in a finite region on the surface so that a large number of energetic knocked-on atoms are created. These knocked-on atoms interact with each other, and then, these are considered to remain as the deformation of lattice in the substrate. Therefore, the yield of displacement by $B_{10}$ remains several times higher than that of $B_{1}$.

\section{Advantages for Shallow Junction Fabrication}

Fig. 4 shows snapshots of $B_{1}$ and spherical $B_{10}$ cluster implantation into Si (001) substrate 8ps after impact. Black spheres represent the implanted B atoms and gray ones are the displaced $\mathrm{Si}$ atoms. Fig. 5 shows the depth profiles of displacements induced by $B_{1}$, horizontal $B_{4}$ and spherical $B_{10}$ cluster. The depth profiles in Fig. 5 are calculated by averaging the results of 50 trials for $B_{1}$ and of 25 trials for $B_{4}$ and $B_{10}$, respectively. Fig. 4 and Fig. 5 indicate that large number of displacements are formed by $B_{10}$ compared with $B_{1}$, as indicated in Fig. 3. Furthermore, $B_{1}$ monomer and $B_{10}$ clusters show a difference in the distribution of displacements. In the case of $B_{1}$ implantation, transient displacements are formed along the trajectory of incident atom, however, these displacements easily recovers because the deposited energy through interaction with incident atom is small. In this case, knocked-on displacements reside around the incident B atom, which is termed 'end-of-range' damage [13] as shown in Fig. 4. The end-of-range displacement is also statistically observed in the region deeper than $30 \AA$ in Fig. 5, which shows larger ratio of displacements for $B_{1}$ compared to those of $B$ clusters. The end-of-range displacements tend to kick-out the boron 


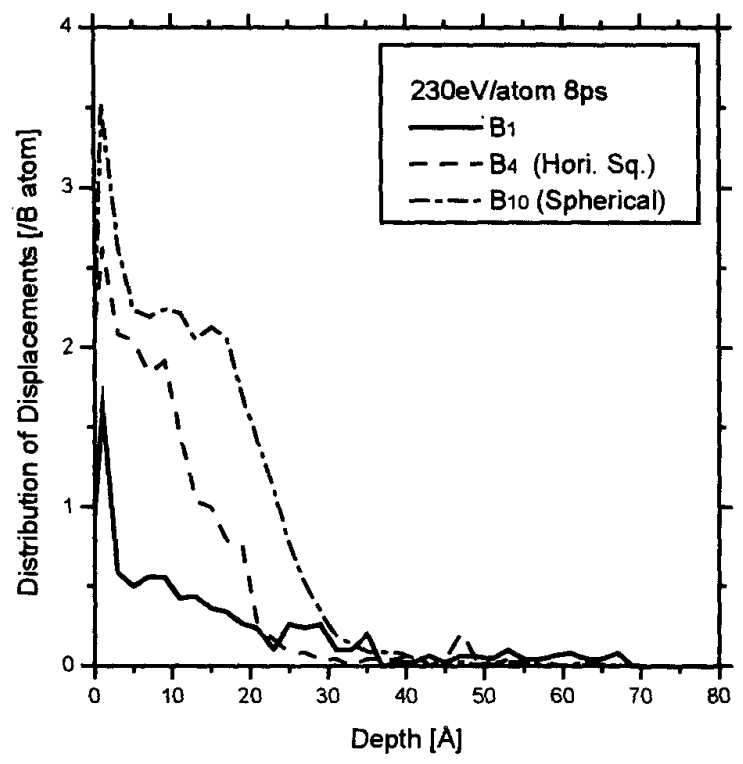

Fig. 5: Depth profile of displacements induced by $B_{1}$ monomer, horizontal $B_{4}$ square and spherical $B_{10}$ clusters 8ps after impact.

atom from a lattice site through annealing and, therefore, the kicked-out $B$ atom diffuses into the deeper region of substrate. This diffusion mechanism, called 'Transient Enhanced Diffusion (TED)' is a serious problem in high-quality shallow junction formation using conventional monomer ion implantation techniques $[4,13]$. On the other hand, $B_{10}$ cluster creates a high density of displacements on the surface of the impact point because of the high-density energy irradiation effect. This region damaged by $B_{10}$ is considered to be amorphized and shows a box-like shape from the surface to the depth of $20 \AA$, which is comparable with the mean implant depth of the $B$ atoms. This characteristic damage formation by $B_{10}$ is expected to result in great advantages in shallow junction formation because of reducing TED without preamorphization process. Through annealing, the reconstruction of a substrate irradiated with $B_{10}$ clusters proceeds from the bottom of the amorphized layer to the surface of the substrate. Therefore, the interstitial $\mathrm{Si}$ atom tends to move to the top surface thus avoiding $B$ atom diffusion into deeper regions of the substrate. It has been observed experimentally that lowenergy $\mathrm{B}_{10} \mathrm{H}_{14}$ implantation into a Si substrate does not cause TED $[6,14]$, in a similar manner to the $B$ implantation into a well pre-amorphized Si substrate.

\section{CONCLIISIONS}

In order to examine the advantages of cluster ion implantation for high-quality shallow junction formation, molecular dynamics simulations of $\mathrm{B}_{1}, \mathrm{~B}_{4}$ and $\mathrm{B}_{10}$ implanting into the $\mathrm{Si}$ substrate were performed. It was found that $B_{10}$ and $B_{4}$ are equivalent to $1 / 10$ and $1 / 4$ low-energy ion implantation, respectively. Each $B$ atoms in the $B_{4}$ and $B_{10}$ clusters act individually and both $B$ types of implantation show similar implant efficiency and implant profiles to that of $B_{1}$, except for the impact of the chain-like structure in orientation perpendicular to the substrate. The enhancement of implant efficiency was shown through the impact of vertical chain-like clusters, whose size is larger than four because of coherency of incident velocity. For each case of impact, the number of displaced $\mathrm{Si}$ atoms reaches the same maximum value, but decays with different speed. The similarity in the maximum number of displacements is caused by the individuality of the incident $B$ atoms, that is, the kinetic energy of each $B$ atoms is transported to the displacements individually without overlapping. The decay speed is lower as the cluster size is larger because of the highdensity energy irradiation effect. $B_{10}$ deposits 10 times higher energy in finite region on the surface compared to $B_{1}$ and creates many energetic knocked-on Si atoms. These energetic substrate atoms remain as displacements on near surface region of the impact point. Therefore, the impact point is well-amorphized by cluster ion impact and this damage region is expected to stop TED of boron atoms to deeper regions of the substrate. These MD results suggests that the cluster ion implantation technique using $\mathrm{B}_{10} \mathrm{H}_{14}$ has great advantages for high-quality shallow junction formation through of low-energy implantation and by suppressing TED as a result of high-yield damage formation.

\section{REFERENCES}

[1] "National Technology road Map for Semiconductors", Semiconductor Industry Association, 1997.

[2] M.I.Current, D.Lopes, M.A.Foad, J.G.England, C.Jones and D.Su, J. Vac. Sci. Technol. B16(1), pp. 327-333, 1998

[3] S.Moffatt, Nucl. Instr. and Meth., B96, p. 1, 1995

[4] H.U.Jäger, J. Appl. Phys. 78(1), pp. 176-186, 1995

[5] K.Goto et al, IEEE Tech. Dig, pp. 471-474, 1997

[6] K.Goto et al, IEEE Tech. Dig. pp. 435-438, 1996

[7] T.Aoki, J.Matsuo, Z.Insepov and I.Yamada, Nucl. Instrum. \& Meth., B121, pp. 49-52, 1997.

[8] T.Seki, T.Kaneko, D.Tkauchi, T.Aoki, J.Matsuo, Z.Insepov and I.Yamada, Nucl. Instrum. \& Meth., B121, pp. 498-502, 1997.

[9] T.Aoki, T.Seki, J.Matsuo, Z.Insepov and I.Yamada, Mats. Chem. \& Phys., 54(1-3), pp. 139-142, 1998.

[10] F.H.Stillinger and T.A.Weber, Phys. Rev. B, B31, p. 5652, 1985.

[11] J.F.Ziegler, J.P.Biersack and U.Littmark, "The stopping and range of ions in solids", Pergamon Press, New York, 1985, p.321.

[12] M.J.Caturla, T.diaz de la Rubia and GH.Gilmer, Nucl. Instrum. \& Meth B196, p. 1, 1995.

[13] K.S.Jones, P.G.Elliman, M.M.Petravic and P.Kringhoj, Appl. Phys. Lett. $68(22)$, p. 3111,1996

[14] T.Kusaba et al, "The boron diffusion in ultra low-energy ( $<1 \mathrm{keV} / \mathrm{atom})$ decaborane $\left(\mathrm{B}_{10} \mathrm{H}_{14}\right)$ Ion Implantation", in this issue. 